ARTICLE

Received 31 May 2016 | Accepted 14 Dec 2016 | Published 22 Feb 2017

DOI: $10.1038 /$ ncomms14265

OPEN

\title{
Sequence variant at 8q24.21 associates with sciatica caused by lumbar disc herniation
}

Gyda Bjornsdottir', Stefania Benonisdottir ${ }^{1}$, Gardar Sveinbjornsson', Unnur Styrkarsdottir ${ }^{1}$, Gudmar Thorleifsson', G. Bragi Walters ${ }^{1}$, Aron Bjornsson², Ingvar H. Olafsson 2,3, Elfar Ulfarsson², Arnor Vikingsson 4,5, Ragnheidur Hansdottir ${ }^{6}$, Karl O. Karlsson ${ }^{6}$, Thorunn Rafnar ${ }^{1}$, Ingileif Jonsdottir ${ }^{1,3}$, Michael L. Frigge ${ }^{1}$, Augustine Kong1,7, Asmundur Oddsson1, Gisli Masson1, Olafur T. Magnusson1, Tomas Gudbjartsson 3,8, Hreinn Stefansson ${ }^{1}$, Patrick Sulem', Daniel Gudbjartsson ${ }^{1,7}$, Unnur Thorsteinsdottir ${ }^{1,3}$, Thorgeir E. Thorgeirsson ${ }^{1}$ $\&$ Kari Stefansson ${ }^{1,3}$

Lumbar disc herniation (LDH) is common and often debilitating. Microdiscectomy of herniated lumbar discs (LDHsurg) is performed on the most severe cases to resolve the resulting sciatica. Here we perform a genome-wide association study on 4,748 LDHsurg cases and 282,590 population controls and discover 37 highly correlated markers associating with LDHsurg at 8q24.21 (between CCDC26 and GSDMC), represented by rs6651255[C] $\left(\mathrm{OR}=0.81 ; P=5.6 \times 10^{-12}\right)$ with a stronger effect among younger patients than older. As rs6651255[C] also associates with height, we performed a Mendelian randomization analysis using height polygenic risk scores as instruments to estimate the effect of height on LDHsurg risk, and found that the marker's association with LDHsurg is much greater than predicted by its effect on height. In light of presented findings, we speculate that the effect of rs 6651255 on LDHsurg is driven by susceptibility to developing severe and persistent sciatica upon LDH.

\footnotetext{
${ }^{1}$ deCODE Genetics/Amgen, Inc., Reykjavik IS-101, Iceland. ${ }^{2}$ Department of Neurosurgery, Landspitali University Hospital, Reykjavik IS-101, Iceland. ${ }^{3}$ Faculty of Medicine, University of Iceland, Reykjavik IS-101, Iceland. ${ }^{4}$ Department of Medicine, Landspitali, National University Hospital, Reykjavik IS-101, Iceland. ${ }^{5}$ Thraut Fibromyalgia Clinic, Reykjavik IS-101, Iceland. ${ }^{6}$ Department of Dentistry, University of Iceland, Reykjavik IS-101, Iceland. ${ }^{7}$ School of Engineering and Natural Sciences, Science Institute, University of Iceland, Reykjavik IS-101, Iceland. ${ }^{8}$ Department of Surgery, Landspitali University Hospital, Reykjavik IS-101, Iceland. Correspondence and requests for materials should be addressed to K.S. (email: kari.stefansson@decode.is).
} 
S ciatica describes the often debilitating form of neuropathic pain that radiates along the sciatic nerve (radiculopathy) ${ }^{1}$. While sciatica can be of various etiologies, the majority of cases $(85-90 \%)$ are caused by lumbar disc degeneration (LDD) resulting in herniation of disc material from the nucleus pulposus into the epidural space, leading to mechanical and/or chemical insult to affected nerve roots ${ }^{1-3}$.

The pathophysiology of lumbar disc herniation (LDH) is not completely understood ${ }^{4}$. Imaging studies of different populations have shown that LDD starts in early adolescence and affects the majority of people over the course of their life ${ }^{5-7}$. Therefore, drawing a diagnostic line between normal consequences of aging and pathological disc changes remains challenging ${ }^{5}$. Twin studies suggest that genetics strongly influence various radiological signs of LDD with heritability estimated up to $74 \%$ (95\% confidence interval (CI) $64-81 \%)^{8,9}$. However, the search for genetic risk factors has not yielded conclusive results $^{10,11}$. A recent genome-wide association study (GWAS), utilizing a quantitative measure created from radiological signs of LDD (lumbar disc space narrowing and presence of osteophytes) in 4,600 individuals of European descent, uncharacterized for LDD symptoms, identified four single-nucleotide polymorphisms (SNPs) with $P$ between $1.8 \times 10^{-9}$ and $3.3 \times 10^{-8}$ (effect $(\beta)$ between -0.13 and 0.23$)^{12}$.

Although herniated lumbar discs are the most common cause of sciatica, they are also observed in up to $50 \%$ of asymptomatic adults by magnetic resonance imaging ${ }^{13-15}$. Furthermore, about a third of symptomatic LDH resolve within weeks or months regardless of intervention ${ }^{13,16,17}$ with only a fraction (5-10\%) leading to persistent sciatica or radiculopathy requiring surgery $^{18,19}$. Microdiscectomy, a minimally invasive spinal surgery for removal of herniated lumbar disc tissue, is indicated only for individuals with severe, progressive and persistent sciatica symptoms that are consistent with location of the radiologically confirmed herniated $\operatorname{disc}^{13,20}$. These surgical criteria, therefore, define a clear and specific phenotype of sciatica secondary to LDH pathology that we here show associates strongly with a sequence variant at $8 \mathrm{q} 24.21$ between GSDMC and CCDC26. Our results provide novel insights into the biological underpinnings of this painful condition.

\section{Results}

A sequence variant at $8 \mathrm{q} 24.21$ associates with LDHsurg. We performed a GWAS of 4,748 Icelanders who underwent microdiscectomy of a herniated lumbar disc (LDHsurg) and 282,590 population controls. The sample represents $90 \%$ of those undergoing this surgical procedure in Iceland over an 18 year period or from 1997-2015 (Table 1).

We applied genome-wide significance thresholds weighted for variant classes, using the threshold of $P<1.1 \times 10^{-9}$ assigned to variants in the lowest impact functional class ${ }^{21}$ (Methods). Altogether, 37 highly correlated sequence variants $\left(r^{2}\right.$ with top marker $>0.78)$ associating with LDHsurg were identified, located at $8 \mathrm{q} 24.21$ between GSDMC and CCDC26 and spanning $\sim 20 \mathrm{~kb}$ (chr8:129,705,470-129,726,726, hg38) (Fig. 1, Supplementary Fig. 1 and Supplementary Table 1). The most significant association was with $\mathrm{rs} 6651255[\mathrm{C}] \quad(\mathrm{AF}=23 \%$; odds ratio $(\mathrm{OR})=0.81 ; 95 \% \mathrm{CI}=0.77,0.86 ; P=5.6 \times 10^{-12}$, a $\chi^{2}$-test was used to calculate $P$ values for all GWAS associations) (Methods). Conditional analysis showed that the signal of 37 variants is fully accounted for by rs6651255[C] (all adjusted $P \geq 0.15$ ) and no other markers within $\pm 2 \mathrm{Mb}$ reached significance. The effect of rs6651255 on LDHsurg did not differ by gender (male vs female $P=0.85$ ). A significant difference in effects was, however, observed when comparing those who underwent LDHsurg at an earlier age ( $\leq 40$ years) to those aged 41 years and older at first LDHsurg $\left(P=1.8 \times 10^{-3}\right)$ (Table 2$)$.

The LDHsurg signal at 8q24.21 does not associate with cancer. The 8q24.21 region, containing $M Y C$ and $P V T 1$, is known to host a number of variants associating with risk of several cancer types $^{22-25}$. However, that region is about $2 \mathrm{Mb}$ upstream of rs6651255. None of the reported markers for cancer in the 8q24.21 region correlate with the LDHsurg signal. Searching for associations of rs6651255[C] with several common cancers ${ }^{26}$ revealed no significant association once the number of tests had been taken into account. The closest protein coding gene ( $\sim 370 \mathrm{~kb}$ downstream) is GSDMC, a gene initially found by researchers seeking genes upregulated in metastatic mouse melanoma cells (hence also termed $M L Z E)^{27}$. Besides expression in skin epithelium, GSDMC is primarily expressed in trachea and spleen and although its function is largely unknown, as a member of the Gasdermin superfamily, it is thought to be involved in regulation of epithelial cell development, apoptosis, carcinogenesis and immune-related functions ${ }^{28-31}$.

Approximately $30 \mathrm{~kb}$ upstream of rs6651255 is the full-length mRNA encoding CCDC26. Also known as RAM, CCDC26 encodes a retinoic acid-dependent modulator of myeloid differentiation ${ }^{32,33}$. Retinoic acid is involved in regulation of skeletal growth, chondrocyte proliferation and aggrecan (the major proteoglycan component of the intervertebral disc) expression and content ${ }^{34}$. Indeed, CCDC26 was considered a candidate gene in a GWAS of cleft lip showing a strong association with a variant (rs987525) located $\sim 400 \mathrm{~kb}$ upstream of CCDC26 (ref. 35). This cleft lip variant is $800 \mathrm{~kb}$ upstream of rs6651255 $\left(r^{2}=0.00056, D^{\prime}=0.084\right)$ and in the present study showed no association with LDHsurg in the Icelandic sample $(\mathrm{OR}=0.98, P=0.40)$. Including rs987525, there are further four SNPs listed in the GWAS catalogue (URLs) located in or near GSDMC or CCDC26. They are rs4295627 associating with glioma, rs10098310 with monocyte count and rs4733724 and rs6470764 with height. Testing these variants in corresponding Icelandic phenotypes shows that SNPs in this region behave comparably in Icelandic data as in other populations (Supplementary Table 2).

The effects of rs6651255 on LDHsurg and height. Three out of the 37 correlated SNPs representing the LDHsurg signal have

Table 1 | Demographics of cases.

\begin{tabular}{|c|c|c|c|c|}
\hline Phenotype & $\mathbf{N}$ & Age $M$ (s.d.) & $n$ with genotypes & $\%$ with genotypes \\
\hline LDHsurg & 5,288 & $44.9(13.7)$ & 4,748 & 89.8 \\
\hline Males & 3,061 & $44.3(13.7)$ & 2,762 & 90.2 \\
\hline Females & 2,227 & $45.7(13.7)$ & 1,986 & 89.2 \\
\hline LDHsurg $\leqslant 40$ years & 2,771 & $33.1(6.1)$ & 1,897 & 68.5 \\
\hline
\end{tabular}

GWAS, genome-wide association studies; LDHsurg, herniated lumbar discs.

Total number of cases undergoing microdiscectomy of an LDHsurg in Iceland 1997-2015 (N) and proportion of cases with genotypes included in the GWAS study (n). 
previously been reported to associate with adult human height in large meta-analyses ${ }^{36-38}$ (Supplementary Tables 2 and 3). All are highly correlated with rs6651255 $\left(r^{2}>0.9\right.$ in Iceland). Consistently, rs6651255[C] associating with lower risk of LDHsurg, also associates with less height in the Icelandic data ( $\beta=-0.03$ s.d. (corresponding to $-0.2 \mathrm{~cm}), P=2.2 \times 10^{-5}$; $N=88,835, \chi^{2}$ test). However, neither rs6470764[T] representing rs6651255, nor any of the 16, out of the 37 correlated SNPs of the signal that were tested in the GIANT BMI meta-analysis ${ }^{39}$, associate with BMI (lowest $P$ value of $0.10(\beta=0.007$; s.e. $=0.004$; $N=321,563)$ ).

Consistent with previous epidemiological results ${ }^{40}$, we observed a positive association between adult height and risk of LDHsurg in Icelandic patients $(\mathrm{OR}=1.25$ for $10 \mathrm{~cm}$ increase in height; $P=4.6 \times 10^{-10} ; N_{\text {case }}=1,929 ; N_{\text {control }}=79,883, t$-test $)$ (Methods). To examine to what degree known adult height variants collectively explain the association with LDHsurg, we computed adult height polygenic scores, based on adjusted GWAS scores for 1.7 million markers tested in the GIANT

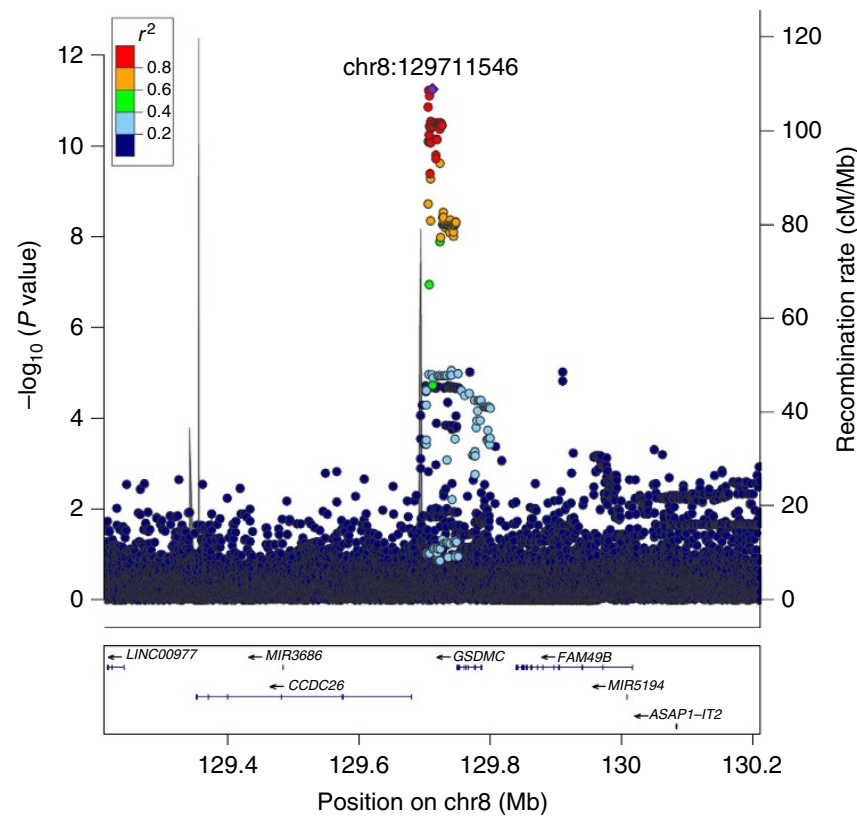

Figure 1 | Regional association plot for the 8q24.21 intergenic locus that associates with undergoing LDHsurg $(N=\mathbf{4 , 7 4 8 )}$. $P$ values $(-\log 10)$ of SNP association with LDHsurg in Iceland is plotted against their positions at the $8 q 24.21$ locus. SNPs are coloured to reflect their LD with rs6651255 (chr8:129711546) (purple diamond) in the data set. The right $y$ axis shows calculated recombination rates at the chromosomal location, plotted as solid grey lines. Known genes in the region are shown underneath the plot, taken from the UCSC genes track in the UCSC Genome Browser. All positions are in in NCBI Build 38 coordinates. A $\chi 2$-test was used to calculate $P$ values. study $^{37}$ (excluding Icelandic data), for Icelanders who had been genotyped with various Illumina SNP chips (Methods). In our data the adult height polygenic scores significantly associate with LDHsurg case-control status $(\mathrm{OR}=1.36$ for $10 \mathrm{~cm}$ increase in height; $P=8.4 \times 10^{-6} ; N_{\text {case }}=3,085 ; N_{\text {control }}=132,822, t$-test $)$.

Given that adult height variants associate collectively with LDHsurg and a SNP corresponding to the LDHsurg signal (rs4733724, Supplementary Table 3) was among the 697 adult height variants reported by the GIANT meta-analysis ${ }^{37}$, we tested whether the other 696 GIANT variants associate independently with LDHsurg (Fig. 2, Methods). While Fig. 2 demonstrates a small positive correlation $\left(R^{2}=0.0072, P=0.026, t\right.$-test) between effects of these GIANT variants on height and logarithm of their LDHsurg risk $(\log \mathrm{OR})$, none of the other GIANT variants significantly associate with LDHsurg at a threshold of $7.2 \times 10^{-5}$ $(P<0.05 / 697)$. The SNP representing our top marker is a clear outlier (Fig. 2). Using the slope from the correlation plot in Fig. 2, the predicted effect of rs6651255[C] on LDHsurg, through its effect on height, would result in an odds ratio of 0.99 , showing that the LDHsurg effect is not proportional to the height effect. These analyses clearly show that the effect of rs6651255 on LDHsurg greatly surpasses that expected due to its effect on height (Fig. 2).

Characterizing rs6651255 effects on related phenotypes. In light of potential structural effects of rs6651255[C] implied by its height association, we also investigated the variant's effect on available phenotypes relating to vertebral bone structure in Icelandic data ${ }^{41}$. The variant did not associate with osteoarthritis of the spine $(\mathrm{OR}=0.96 ; P=0.35)$, bone mineral density of the spine $(\beta=-0.01 ; P=0.31)$, or osteoporotic vertebral fractures $(\mathrm{OR}=1.02 ; P=0.64)$ (Supplementary Table 4$)$.

As coded radiological reports for this large sample of LDHsurg cases were unavailable for the study, we tested all published markers $\left(P<10^{-5}\right)$ from the previously mentioned GWAS of radiologically defined LDD (a quantitative trait phenotype based on lumbar disc space narrowing and osteophytes growth $)^{12}$ for association with our LDHsurg phenotype. None of the 44 markers associates with risk of undergoing LDHsurg $\left(P<0.05 / 44=1.1 \times 10^{-3}\right.$, Supplementary Table 5).

To further characterize the effects of rs6651255, we identified an independent sample of Icelandic cases from hospital records $\left(N_{\text {case }}=930\right)$ diagnosed as the LDHsurg cases, (with intervertebral disc disorder with radiculopathy represented by ICD-10 code M51.1) who, however, had no history of LDHsurg. This indicating that their associated sciatica symptoms resolved over time and did not become severe enough to warrant surgery. There was no association observed with rs6651255 in this sample $(\mathrm{OR}=0.95 ; P=0.50)$.

Taken together, these findings suggest that the effect of rs6651255 on risk of undergoing LDHsurg is driven, not primarily by the morphology associated with herniated lumbar discs, but rather by the severity and persistence of associated

Table 2 | GWAS results for variant rs6651255[C] in LDHsurg in Iceland.

\begin{tabular}{|c|c|c|c|c|}
\hline Phenotype & $\mathbf{N}$ & OR & $95 \% \mathrm{Cl}$ & $\boldsymbol{P}$ \\
\hline LDHsurg & 4,748 & 0.81 & $(0.77,0.86)$ & $5.61 \times 10^{-12}$ \\
\hline LDHsurg $\leqslant 40 y r{ }^{\star}$ & 1,898 & 0.72 & $(0.65,0.79)$ & $3.20 \times 10^{-11}$ \\
\hline LDHsurg $>40 y r{ }^{\star}$ & 2,850 & 0.87 & $(0.81,0.93)$ & $9.63 \times 10^{-5}$ \\
\hline
\end{tabular}




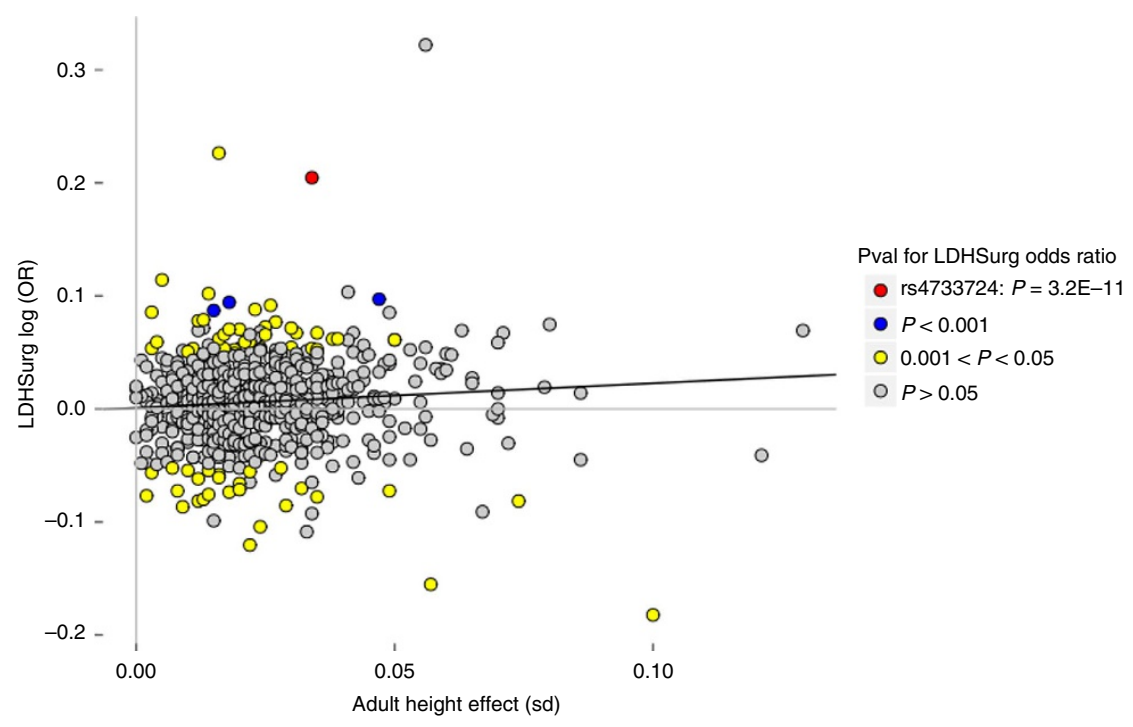

Figure 2 | Correlation of GIANT variants' effect on height and risk of LDHsurg. Scatter plot showing how the 689 out of 697 adult height variants reported by GIANT ${ }^{37}$ that we have LDHsurg association results for, affect adult height ( $x$ axis) and LDHsurg ( $y$ axis) in our data. The effect allele (minor or major) of each variant was concluded to be the one that has a positive effect on adult height in our data. The black solid line, $y=0.0010+0.22 x+\mathrm{e}$, represents weighted linear regression, excluding rs 4733724 , with $R^{2}=0.0072$ and $P=0.026$, using MAF(1-MAF) as weights. A $t$-test was used to calculate $P$ value.

sciatica. We therefore tested whether rs6651255[C] associated with chronic or neuropathic pain in independent Icelandic samples with fibromyalgia $\left(N_{\text {case }}=2,142\right)$, temporomandibular joint disorder (TMD, $\left.N_{\text {case }}=1,455\right)$ and migraine $\left(N_{\text {case }}=3,816\right)$, in addition to a small sample with neuropathic pain as determined by the DN4 screening questionnaire $\left(N_{\text {case }}=394\right)$ (Methods). No significant associations with rs6651255 were detected. As larger sample sizes are needed to reach definite conclusions we provide the association results (Supplementary Table 6) to facilitate future meta-analysis efforts.

Effect of rs6651255 on expression of nearby genes. Finally, to assess the potential effect of rs6651255 on gene expression (eQTL) we examined data from the Genotype-Tissue Expression (GTEx) project (URLs) available from multiple tissues, however, not including tissue from the intervertebral disc. In GTEx data, rs6651255 has a significant eQTL (cis window defined as $\pm 1 \mathrm{Mb}$ around gene transcript start site, false discovery rate $<0.05)$ with GSDMC in two tissues; esophagus-mucosa $\left(P=9.2 \times 10^{-14}\right)$ and Sun-exposed skin of lower leg $\left(P=4.5 \times 10^{-10}\right)$, while association with CCDC26 (RP11-274M4.1) was only present in esophagus-mucosa $\left(P=3.7 \times 10^{-6}\right)$ (Supplementary Table 7$)$. However, the best LDHsurg association signal (represented by rs6651255) and the best eQTL in the same tissues do not coincide. The variant rs4345520 has the most significant eQTL with GSDMC (esophagus-mucosa, $P=3.0 \times 10^{-42}$; Skin-Sun-exposed (lower leg), $P=3.3 \times 10^{-28}$ ) and rs10092783 the most significant eQTL with CCDC26 (RP11-274M4.1) (esophagus-mucosa, $\left.P=6.7 \times 10^{-16}\right)$. These two variants are highly correlated $\left(r^{2}=0.82\right)$, while they are moderately correlated with rs6651255 (all $r^{2}<0.29$ ). (Supplementary Table 7). In addition, a cis-eQTL analysis was performed in the Icelandic data based on RNA sequencing of white blood cells $(N=1,002)$ and adipose tissue $(N=673)$ samples. We assessed associations with probes from the five genes within $\pm 500 \mathrm{~Kb}$ of rs6651255 (Fig. 1). At a significance threshold of $1.0 \times 10^{-2}(P<0.05 / 5)$ we did not observe associations of rs6651255 with gene expression in white blood cells or adipose tissue.

\section{Discussion}

We have discovered an association between common, correlated, intergenic sequence variants and risk of undergoing microdiscectomy of a herniated lumbar disc (LDHsurg); a spinal surgery performed to alleviate persistent and painful sciatica symptoms. The signal is represented by rs6651255[C] that also associates with less height, potentially reflecting structural effects involved in the pathogenesis of LDH. However, our results show that this variant does not affect risk of LDHsurg through height (Fig. 2). In addition, rs6651255 does not affect the skeletal phenotypes that we tested; osteoarthritis of the spine, bone mineral density of the spine or vertebral osteoporosis, nor did we find associations between markers from the only identified GWAS of morphological changes associated with LDD (albeit only radiologically defined without symptom characterization $)^{12}$. Indeed, clinical studies have established that there is an unclear relationship between radiological signs of $\mathrm{LDH}$ and degree of sciatica or other clinical symptoms ${ }^{15}$. In keeping with the main indication for LDHsurg ${ }^{13,20}$; the neuropathic and painful symptoms of sciatica, we find of interest that height is inversely correlated with peripheral nerve conduction velocity ${ }^{42}$ and has been shown to increase risk of peripheral sensory neuropathy in diabetes $^{43}$, peripheral insensate neuropathy ${ }^{44}$, HIV neuropathy ${ }^{45}$ and post-mastectomy neuropathic pain syndrome ${ }^{46}$. While the pathogenesis behind the relationship between height and neuropathic pain is unclear, increased height in humans is known to associate with increased axonal length and greater axon surface area ${ }^{47}$. The weak, but directionally consistent association $(\mathrm{OR}=0.83, P=0.04)$ of rs6651255[C] with neuropathic pain as measured by the DN4 is of interest in this context, but requires further study in larger samples.

When selecting cases for microdiscectomy, neurosurgeons have established that the herniated disc material is likely to cause the associated sciatica symptoms ${ }^{13,20}$. Indeed in most cases, rapid symptom relief follows microdiscectomy, but in up to $20 \%$ of cases symptoms persist after surgery ${ }^{13,18,48,49}$. Besides the symptoms caused by mechanical compression on nerve roots by the herniated lumbar disc, inflammatory and immunological consequences are also considered a factor in sciatica symptom 
severity and duration ${ }^{50,51}$. Of interest in this context is that GSDMC, downstream of rs6651255, is highly expressed in the spleen ${ }^{27}$ which plays a significant role in adaptive immune responses ${ }^{52}$. While the role of the immune system in regression of herniated discs and/or development of sciatica is not clear, the immune system is known to become activated by the otherwise immune-privileged nucleus pulposus as it herniates from its protected environment into the immuneregulated epidural space ${ }^{53}$. The subsequent migration of immune cells into the herniated disc tissues and increased expression of inflammatory cytokines is accompanied by the ingrowth of nociceptive nerve fibres into the disc, a process thought to contribute to the pathogenic development of the associated painful symptoms ${ }^{53}$.

The surgically defined phenotype used in this study was selected to represent a severe, symptomatic form of LDD, that is, herniated lumbar disc with sciatica indicating LDHsurg. In light of the clear clinical criteria associated with this type of surgery and the findings presented here, we speculate that the observed effect of rs6651255 on risk of undergoing LDHsurg is mainly driven by the severity of symptoms, that is, susceptibility to developing persistent and painful sciatica in the presence of the mechanical and biochemical nerve root insult created by a herniated lumbar disc. While our findings await replication in a comparable sample, we have provided compelling evidence for this variant having a significant role in the development of sciatica resulting from a herniated lumbar disc. The observed association in the Icelandic population is highly significant and we were able to exclude that the effect on sciatica is solely through the variant's effect on height. However, it is clear that further studies are required to complete this complicated but intriguing picture.

\section{Methods}

Study populations and phenotypes. Data on microsurgical excision of herniated lumbar intervertebral discs (LDHsurg) were obtained from the Landspitali National University Hospital (Landspitali) databases of surgical procedures (that is, lumbar microdiscectomy with surgical code ABC16 according to the NOMESCO (Nordic Medico-Statistical Committee) system) ${ }^{54}$. The so-defined phenotype is represented in the current paper by 'LDHsurg'. Demographics of the defined sample are presented in Table 1 . Landspitali is a tertiary reference centre hospital and the only hospital in Iceland offering neurosurgery. Microdiscectomy consists of removing a portion of the intervertebral disc, the herniated or protruding portion that is compressing the traversing spinal nerve root, by way of a small incision and using a surgical microscope. This well-established technique has become the most common surgery performed by neurosurgeons in Iceland and elsewhere ${ }^{13,20}$. It was first performed in Landspitali in 1981 and has since become standard treatment for herniated lumbar discs with persistent, painful sciatica or radiculopathy that does not respond to conservative treatment ${ }^{20}$. The data used in the present study cover microdiscectomy surgeries performed in the hospital's Department of Neurosurgery over a period of 18 years (1997-2015). As shown in Table 1, the proportions of genotyped cases out of the total cases undergoing microdiscectomy surgery during this period support non-bias in the discovery phenotype. Data on individuals diagnosed with herniated intervertebral discs with radiculopathy (ICD-10 code M51.1) who did not undergo microdiscectomy (LDHsurg), were obtained from the Landspitali database of discharge diagnoses (for the same time period as LDHsurg, or 1997-2015). During this period, M51.1 was the most prevalent hospital diagnosis preceding LDHsurg, with a total of 6,280 individuals with this diagnosis. The 5,288 individuals who underwent LDHsurg (Table 1) were excluded as were those diagnosed with M51.1 within 6 months of the end of the study period in 2015, to ascertain that we did not include newly diagnosed cases that would within 6 months become candidates for LDHsurg (according to clinical guidelines in Iceland, LDHsurg is performed if symptoms progress and/or severity persists for $2-3$ months). Age and gender distribution of this sample of confirmed LDH without LDHsurg was comparable to the larger LDHsurg sample (Supplementary Table 8).

Height measurements from 88,835 Icelanders were collected in deCODE's resident assessment instrument studies. Height $(\mathrm{cm})$ was either measured using a stadiometer with the subjects wearing no shoes or self-reported on questionnaires by individuals. Adult height measurements were corrected for year of birth and standardized separately for each of the sexes to have a standard normal distribution. Measured and self-reported heights were corrected separately. Height measurements were available for 1929 LDHsurg cases and 79,886 controls.
Information on vertebral osteoarthritis and bone mineral density was obtained from the Landspitali electronic health records. Secondary osteoarthritis and post-trauma osteoarthritis were excluded from the list. Bone densitometry (DEXA, Hologic QDR4500A) was also obtained from Landspitali ${ }^{41}$. Bone mineral density of the spine (L2-L4) was age and weight corrected and standardized in each gender separately. Vertebral fractures were self-reported in a detailed questionnaire of osteoporosis related information, using additional hospital data to increase classification accuracy, for example, by excluding high-trauma fractures, corticosteroid users, and women with early menopause from the fracture list ${ }^{41}$

The Icelandic migraine sample was recruited from various sources including diagnoses from neurologists and self-reported migraine according to a validated questionnaire as has been described in detail elsewhere ${ }^{55}$. Fibromyalgia cases were characterized by a rheumatologist heading the only clinic in Iceland specializing in diagnosis and treatment of fibromyalgia. All patients were diagnosed according to the 2010 American College of Rheumatology (ACR) criteria ${ }^{56}$. Data on TMD were obtained via the dental department at the University of Iceland. All patients were diagnosed by dentists specializing in TMD following the diagnostic criteria for temporomandibular disorders (DC/TMD) ${ }^{57}$. Patients with a history of TMD secondary to trauma were excluded. Participants in the deCODE pain projects who answered the DN4 screening questionnaire for neuropathic pain ${ }^{58}$ were recruited on the basis of having various chronic pain conditions (chronic pain defined as lasting 3 months or longer) in addition to post-surgical patients who by the nature of their surgery had sustained neural lesions. On the basis of studies evaluating sensitivity and specificity of DN4 scores compared with clinical diagnoses of neuropathic pain, those who scored 4 and above on the DN4 scale of $0-7$ were classified as having neuropathic pain ${ }^{58}$

All samples and questionnaire data were collected through studies approved by the National Bioethics Committee and the Data Protection Authority in Iceland. Data were analysed within the context of a study on the genetics of chronic and neuropathic pain with permission from the National Bioethics Committee (VSNb2012090009/03.12) and overseen by the Data Protection Authority. All participants signed informed consent before blood samples were drawn. All personal identifiers were encrypted by a third-party system overseen by the Icelandic Data Protection Authority ${ }^{59}$. The association studies were performed using genotypes from these data in addition to whole-genome sequence data and imputation approaches previously outlined ${ }^{60}$ and further described below.

Genotyping and imputation. Genotyping and imputation methods and the association analysis method in the Icelandic samples are previously described ${ }^{60}$ with some modifications described here. The whole genomes of 8,453 Icelanders were sequenced using Illumina technology to a mean depth of at least $\times 10$ $($ median $\times 32$ ). SNPs and indels were identified and genotypes called using joint calling with the Genome Analysis Toolkit HaplotypeCaller (GATK version 3.3.0) (ref. 61). The sequence variants identified in the 8,453 sequenced Icelanders were then imputed into 150,656 Icelanders who had been genotyped with various Illumina SNP arrays and their genotypes phased using long-range phasing ${ }^{60}$. Using familial imputation ${ }^{60}$, the sequence variants were imputed into untyped relatives of the array-typed to further increase the sample size for association analysis and increased the power to detect associations. Individuals who had undergone LDHsurg were either genotyped individuals $(N=3,097)$ or untyped first and second degree relatives of genotyped individuals $(N=1,651)$. The group of controls consisted of array-typed individuals $(N=132,966)$ and untyped first and second degree relatives $(N=149,624)$; resulting in 282,590 population controls. A total of 21.6 million variants were used in the association analysis under an additive model All of the tested variants had imputation information over 0.85 and met quality control standards ${ }^{60}$.

\section{Sample preparation and DNA whole-genome sequencing methods. Our} dataset contains samples obtained using three different library preparation methods from Illumina. In addition sequencing was performed using three different types of Illumina sequencing instruments.

(a) Standard TruSeq DNA library preparation method. Illumina GAIIx and/or HiSeq 2000 sequencers.

(b) TruSeq DNA PCR-free library preparation method. Illumina HiSeq 2500 sequencers.

(c) TruSeq Nano DNA library preparation method. Illumina HiSeq X sequencers.

The Standard TruSeq DNA library preparation method involves isolating approximately $1 \mu \mathrm{g}$ of genomic DNA from frozen blood samples and fragmenting to a mean target size of $\sim 300-400$ bp using a Covaris E210 instrument. The resulting fragmented DNA was end repaired using T4 and Klenow polymerases and T4 polynucleotide kinase with $10 \mathrm{mM}$ dNTP followed by addition of an 'A' base at the ends using Klenow exo fragment ( $3^{\prime}$ to $5^{\prime}$-exo minus) and dATP $(1 \mathrm{mM})$. Sequencing adaptors containing ' $\mathrm{T}$ ' overhangs were ligated to the DNA products followed by agarose (2\%) gel electrophoresis. Fragments of $\sim 450-500 \mathrm{bp}$ were isolated from the gels (QIAGEN Gel Extraction Kit), and the adaptormodified DNA fragments were PCR enriched for ten cycles using Phusion DNA polymerase (Finnzymes Oy) and a PCR primer cocktail (Illumina) needed for 
paired-end sequencing. Enriched libraries were purified using AMPure XP beads. The quality and concentration of the libraries were assessed with the Agilent 2100 Bioanalyzer using the DNA 1000 LabChip. Libraries were stored at $-20^{\circ} \mathrm{C}$. Sequencing-by-synthesis (SBS) was performed on either Illumina $\mathrm{GAII}_{\mathrm{x}}$ or HiSeq 2000 instruments, respectively. Paired-end libraries were sequenced using $2 \times 76,2 \times 101$ or $2 \times 120$ cycles of incorporation and imaging with Illumina SBS kits, TruSeq v5 for the GAIIx. For the HiSeq 2000, $2 \times 101$ cycles with SBS kits v2.5 or v3 were employed. Each library was initially run on a single lane on a $\mathrm{GAII}_{\mathrm{x}}$ for validation, assessing optimal cluster densities, insert size, duplication rates and comparison with chip genotyping data. Following validation, the desired sequencing depth $(\times 10$ to $\times 30)$ was then obtained using either sequencing platform. Targeted raw cluster densities ranged from $500-800 \mathrm{~K}^{-1}$ $\mathrm{mm}^{-2}$, depending on the version of both the sequencing chemistry and the data imaging/analysis software packages (SCS.2.8/RTA1.8 or SCS2.9/RTA1.9 for the GAIIx and HCS1.3.8. or HCS1.4.8 for HiSeq 2000). Real-time analysis involved conversion of image data to base calling in real-time.

Using the TruSeq DNA PCR-free library preparation method, paired-end libraries for sequencing were prepared according to the manufacturer's instructions (Illumina, TruSeq DNA PCR-free). In short, $\sim 1 \mu \mathrm{g}$ of genomic DNA, isolated from frozen blood samples, was fragmented to a mean target size of $350 \mathrm{bp}$ using a Covaris E210 ultrasonicator followed by clean-up using AmPure $\mathrm{XP}$ purification beads. Blunt-end DNA from the resulting fragments was generated using a mix of $3^{\prime}>5^{\prime}$ exonuclease and $5^{\prime}>3^{\prime}$ polymerase activities, respectively, followed by $5^{\prime}$-phosphorylation using T4 polynucleotide kinase. Size-selection of the blunt-end fragments was done using a two-step purification strategy with different ratios of the AmPure XP purification beads $(\times 0.6$ and $\times 1)$. Finally, $3^{\prime}$-adenylation and ligation of barcoded adapters was performed, followed by cleanup with magnetic beads. The quality and concentration of the libraries were assessed with the Agilent 2100 Bioanalyzer using the DNA 1000 LabChip (Agilent). Barcoded libraries were stored at $-20^{\circ} \mathrm{C}$. All steps in the workflow were monitored using an in-house laboratory information management system with barcode tracking of all samples and reagents. All samples were first pooled (12-24 plex) and sequenced on Illumina's MiSeq instruments $(2 \times 25$ cycles) to assess quality and effective concentration of sequencing libraries. Subsequent deep sequencing was done on HiSeq 2500 instruments, were each sample was sequenced on three lanes, generating $>100 \mathrm{~Gb}$ of raw data and at least $30 \mathrm{X}$ coverage. Sequencing was done using TruSeq v3 reagents, paired-end $2 \times 100$ cycles. System operation and base calling in real-time was done using HCS 2.2.38 and RTA 1.18.61.

Finally, the TruSeq Nano DNA library preparation method involves sample preparation and sequencing using a method that is essentially the same as described above for the TruSeq DNA PCR-free method, except the input amount was $100 \mathrm{ng}$ of genomic DNA (instead of $1 \mu \mathrm{g}$ ) and following clean-up of adapter ligated DNA, the samples were enriched by eight-cycles of PCR using a PCR primer cocktail, followed by Ampure XP bead clean-up. The quality and concentration of the libraries were assessed with the Perkin Elmer LabChip GX instrument using the HT DNA HiSens reagent kit. Sequencing was done using the HiSeq X HD reagent kit. Each sample was loaded onto the HiSeq X instrument at a concentration of $300 \mathrm{pM}$ and sequenced to high depth $(>30 \times)$. System operation and base calling in real-time was done using HCSX 3.1.26 and RTA2 2.3.9.

Association analysis. Association testing for case-control analysis was performed using logistic regression, adjusting for gender, age and county. A generalized form of linear regression was used to test for association of quantitative traits with SNPs.

Our method of testing for association takes the closest relatedness into account using a mixed effect model ${ }^{60}$. To further account for inflation in test statistics due to relatedness and stratification, we applied the method of linkage disequilibrium (LD) score regression ${ }^{62}$. With a set of $1.1 \mathrm{M}$ variants we regressed the $\chi^{2}$ statistics from our GWAS scan against LD score and used the intercept as a correction factor. The LD scores were downloaded from an LD score database (URL's). The estimated correction factor was 1.15 for LDHsurg, 1.05 for LDHsurg $\leq 40 \mathrm{yrs}$, 1.08 for LDHsurg > 40yrs, 1.08 for LDHsurg males and 1.06 for LDHsurg females. The correction factor was 1.48 for adult height, 1.07 for osteoporosis with vertebral fractures, 1.44 for osteoarthritis of the spine, 1.14 for bone mineral density of spine, 1.01 for LDH (ICD-10 code M51.1) excluding LDHsurg, 1.09 for TMD, 1.11 for fibromyalgia, 1.51 for migraine and 1.04 for DN4-determined neuropathic pain.

The threshold for genome-wide significance was corrected for multiple testing with a weighted Bonferroni adjustment using as weights the enrichment of variant classes with predicted functional impact among association signals ${ }^{21}$. With $21,568,490$ sequence variants being tested, the weights given in the study by Sveinbjornsson et al. ${ }^{21}$ were rescaled to control the family-wise error rate. This yielded significance thresholds of $3.7 \times 10^{-7}$ for high-impact variants $(N=6,308)$, $7.4 \times 10^{-8}$ for moderate-impact variants $(N=121,793), 5.3 \times 10^{-9}$ for low-impact variants $(N=1,329,163), 3.4 \times 10^{-9}$ for other DNase I hypersensitivity sites (DHS) variants $(N=3,262,787)$ and $1.1 \times 10^{-9}$ for other non-DHS variants $(N=16,848,439)$.

In our data, 37 variants, all located at chr8:129,705,470-129,726,726 (hg38), associated significantly with LDHsurg. We performed conditional analysis for all variants with info $>0.9$ that are positioned within $\pm 2 \mathrm{Mb}$ of these 37 variants, with rs6651255[C] the variant with the most significant association, as a covariate. No variants in the area had a significant adjusted $P$ value (all adjusted $P>2.3 \times 10^{-4}$ ).

Logistic regression was performed for LDHsurg case-control status, adjusting for gender and county of origin, with height in terms of SD in $\mathrm{cm}$ as explanatory variable $\left(N_{\text {case }}=1,929 ; N_{\text {control }}=79,883\right)$.

To generate height polygenic risk scores, GWAS results for 2,550,858 variants were computed based on GIANT ${ }^{37}$ data after having removed Icelandic samples. Imputed genetic variants were available for 3,085 adult LDHsurg cases and 132,822 controls. Of the GWAS variants which were also available in the imputation set, 1,746,527 were biallelic SNPs and met quality control standards. LDpred $^{63}$ was used to adjust for the correlation among the effects of these variants due to linkage disequilibrium and these adjusted effects were used to produce the adult height polygenic score. Logistic regression was performed for LDHsurg case-control status, adjusting for gender and county of origin, with the polygenic score as explanatory variable. To put the odds ratios for one unit increase in polygenic score in terms of increase in ten $\mathrm{cm}$, we ran a linear regression for adult height in terms of $\mathrm{SD}$ in $\mathrm{cm}$ with the polygenic score as explanatory variable $(N=80,546)$. The regression slope for the polygenic score $(\beta=5.5$, $P<1 \times 10^{-300}$ ) was then used to estimate how many units of the polygenic score would increase height by $10 \mathrm{~cm}(1.8=10 / 5.5$ units $)$.

Data availability. Data supporting the findings of this study are available within the article and its Supplementary Information files. Summary level data of markers tested for association is described in Scientific data as of 2015 with the identifier http://dx.doi.org/10.1038/sdata.2015.11 (ref. 64). Whole-genome sequencing summary data are available at the European Variant Archive (EVA, https://www.ebi.ac.uk/eva/) under the accession code PRJEB8636.

GTEx data (http://www.gtexportal.org) accessed 8 August 2016; LD Score Database (ftp://atguftp.mgh.harvard.edu/brendan/

1k_eur_r2_hm3snps_se_weights.RDS) accessed 23 June 2015; GWAS catalogue (https://www.ebi.ac.u/gwas/home) accessed 8 September 2016.

\section{References}

1. Ropper, A. H. \& Zafonte, R. D. Sciatica. N. Engl. J. Med. 372, 1240-1248 (2015).

2. Valat, J. P., Genevay, S., Marty, M., Rozenberg, S. \& Koes, B. Sciatica. Best Pract. Res. Clin. Rheumatol. 24, 241-252 (2010).

3. Mixter, W. J. \& Barr, J. S. Rupture of the intervertebral disc with involvement of the spinal canal. N. Engl. J. Med. 211, 210-215 (1934).

4. Winkelstein, B. A., Allen, K. D. \& Setton, L. A. in The Intervertebral Disc (eds Shapiro, L. M. \& Risbud, M. V.) 305-326 (Springer-Verlag, Wien, 2014).

5. Kjaer, P., Leboeuf-Yde, C., Korsholm, L., Sorensen, J. S. \& Bendix, T. Magnetic resonance imaging and low back pain in adults: a diagnostic imaging study of 40-year-old men and women. Spine (Phila $\mathrm{Pa}$ 1976) 30, 1173-1180 (2005).

6. Kjaer, P., Leboeuf-Yde, C., Sorensen, J. S. \& Bendix, T. An epidemiologic study of MRI and low back pain in 13-year-old children. Spine (Phila Pa 1976) 30, 798-806 (2005).

7. Cheung, K. M. et al. Prevalence and pattern of lumbar magnetic resonance imaging changes in a population study of one thousand forty-three individuals. Spine (Phila Pa 1976) 34, 934-940 (2009).

8. Sambrook, P. N., MacGregor, A. J. \& Spector, T. D. Genetic influences on cervical and lumbar disc degeneration: a magnetic resonance imaging study in twins. Arthritis Rheum. 42, 366-372 (1999).

9. Battie, M. C. et al. Similarities in degenerative findings on magnetic resonance images of the lumbar spines of identical twins. J. Bone Joint Surg. Am. 77, 1662-1670 (1995).

10. Battie, M. C. \& Videman, T. Lumbar disc degeneration: epidemiology and genetics. J. Bone Joint Surg. Am. 88(Suppl 2): 3-9 (2006).

11. Eskola, P. J. et al. Genetic association studies in lumbar disc degeneration: a systematic review. PLoS ONE 7, e49995 (2012).

12. Williams, F. M. et al. Novel genetic variants associated with lumbar disc degeneration in northern Europeans: a meta-analysis of 4600 subjects. Ann. Rheum. Dis. 72, 1141-1148 (2013).

13. Kreiner, D. S. et al. An evidence-based clinical guideline for the diagnosis and treatment of lumbar disc herniation with radiculopathy. Spine J. 14, 180-191 (2014).

14. Brinjikji, W. et al. Systematic literature review of imaging features of spinal degeneration in asymptomatic populations. Am. J. Neuroradiol. 36, 811-816 (2015).

15. Haldeman, S. North American Spine Society: failure of the pathology model to predict back pain. Spine (Phila Pa 1976) 15, 718-724 (1990).

16. Atlas, S. J., Keller, R. B., Wu, Y. A., Deyo, R. A. \& Singer, D. E. Long-term outcomes of surgical and nonsurgical management of sciatica secondary to a lumbar disc herniation: 10 year results from the maine lumbar spine study. Spine (Phila $\mathrm{Pa}$ 1976) 30, 927-935 (2005). 
17. Soliman, J. et al. Limited microdiscectomy for lumbar disk herniation: a retrospective long-term outcome analysis. J. Spinal Disord. Tech. 27, E8-E13 (2014).

18. Artus, M., van der Windt, D., Jordan, K. P. \& Croft, P. R. The clinical course of low back pain: a meta-analysis comparing outcomes in randomised clinical trials (RCTs) and observational studies. BMC Musculoskelet. Disord. 15, 68 (2014).

19. Konstantinou, K. \& Dunn, K. M. Sciatica: review of epidemiological studies and prevalence estimates. Spine (Phila $\mathrm{Pa}$ 1976) 33, 2464-2472 (2008).

20. Jensdottir, M., Gudmundsson, K., Hannesson, B. \& Gudmundsson, G. 20 years follow-up after the first microsurgical lumbar discectomies in Iceland. Acta Neurochir. (Wien) 149, 51-58 (2007).

21. Sveinbjornsson, G. et al. Weighting sequence variants based on their annotation increases power of whole-genome association studies. Nat. Genet. 48, 314-317 (2016).

22. Tseng, Y. Y. et al. PVT1 dependence in cancer with MYC copy-number increase. Nature 512, 82-86 (2014).

23. Ghoussaini, M. et al. Multiple loci with different cancer specificities within the 8q24 gene desert. J. Natl Cancer. Inst. 100, 962-966 (2008).

24. Amundadottir, L. T. et al. A common variant associated with prostate cancer in European and African populations. Nat. Genet. 38, 652-658 (2006).

25. Gudmundsson, J. et al. A common variant at $8 \mathrm{q} 24.21$ is associated with renal cell cancer. Nat. Commun. 4, 2776 (2013).

26. Stacey, S. N. et al. A germline variant in the TP53 polyadenylation signal confers cancer susceptibility. Nat. Genet. 43, 1098-1103 (2011).

27. Watabe, K. et al. Structure, expression and chromosome mapping of MLZE, a novel gene which is preferentially expressed in metastatic melanoma cells. Jpn J. Cancer Res. 92, 140-151 (2001).

28. Tamura, M. \& Shiroishi, T. GSDM family genes meet autophagy. Biochem. J. 469, e5-e7 (2015)

29. Shi, J. et al. Cleavage of GSDMD by inflammatory caspases determines pyroptotic cell death. Nature 526, 660-665 (2015).

30. Shi, P. et al. Loss of conserved Gsdma3 self-regulation causes autophagy and cell death. Biochem. J. 468, 325-336 (2015).

31. Saeki, N. \& Sasaki, H. in Endothelium and Epithelium (eds Carrasco, J. \& Mota, M.) 193-211 (Nova Science Publishers, Inc., 2012).

32. Yin, W., Rossin, A., Clifford, J. L. \& Gronemeyer, H. Co-resistance to retinoic acid and TRAIL by insertion mutagenesis into RAM. Oncogene 25, 3735-3744 (2006).

33. Hirano, T. et al. Genes encoded within $8 \mathrm{q} 24$ on the amplicon of a large extrachromosomal element are selectively repressed during the terminal differentiation of HL-60 cells. Mutat. Res. 640, 97-106 (2008).

34. Williams, J. A. et al. Retinoic acid receptors are required for skeletal growth, matrix homeostasis and growth plate function in postnatal mouse. Dev. Biol. 328, 315-327 (2009).

35. Birnbaum, S. et al. Key susceptibility locus for nonsyndromic cleft lip with or without cleft palate on chromosome 8q24. Nat. Genet. 41, 473-477 (2009).

36. Lango Allen, H. et al. Hundreds of variants clustered in genomic loci and biological pathways affect human height. Nature 467, 832-838 (2010).

37. Wood, A. R. et al. Defining the role of common variation in the genomic and biological architecture of adult human height. Nat. Genet. 46, 1173-1186 (2014).

38. He, M. et al. Meta-analysis of genome-wide association studies of adult height in East Asians identifies 17 novel loci. Hum. Mol. Genet. 24, 1791-1800 (2015).

39. Locke, A. E. et al. Genetic studies of body mass index yield new insights for obesity biology. Nature 518, 197-206 (2015).

40. Wahlstrom, J., Burstrom, L., Nilsson, T. \& Jarvholm, B. Risk factors for hospitalization due to lumbar disc disease. Spine (Phila Pa 1976) 37, 1334-1339 (2012).

41. Styrkarsdottir, U. et al. Sequence variants in the PTCH1 gene associate with spine bone mineral density and osteoporotic fractures. Nat. Commun. 7, 10129 (2016).

42. Campbell, Jr. W. W., Ward, L. C. \& Swift, T. R. Nerve conduction velocity varies inversely with height. Muscle Nerve 4, 520-523 (1981).

43. Adler, A. I. et al. Risk factors for diabetic peripheral sensory neuropathy. Results of the Seattle Prospective Diabetic Foot Study. Diabetes Care 20, 1162-1167 (1997).

44. Cheng, Y. J. et al. Peripheral insensate neuropathy--a tall problem for US adults? Am. J. Epidemiol. 164, 873-880 (2006).

45. Evans, S. R. et al. Peripheral neuropathy in HIV: prevalence and risk factors. AIDS 25, 919-928 (2011).
46. Smith, W. C., Bourne, D., Squair, J., Phillips, D. O. \& Chambers, W. A. A retrospective cohort study of post mastectomy pain syndrome. Pain 83, 91-95 (1999).

47. Ho, K. C., Gwozdz, J. T., Hause, L. L. \& Antuono, P. G. Correlation of neuronal cell body size in motor cortex and hippocampus with body height, body weight, and axonal length. Int. J. Neurosci. 65, 147-153 (1992).

48. Weinstein, J. N. et al. Surgical vs nonoperative treatment for lumbar disk herniation: the Spine Patient Outcomes Research Trial (SPORT): a randomized trial. JAMA 296, 2441-2450 (2006).

49. Parker, S. L. et al. Incidence of low back pain after lumbar discectomy for herniated disc and its effect on patient-reported outcomes. Clin. Orthop. Relat Res. 473, 1988-1999 (2015).

50. Olmarker, K. et al. Inflammatogenic properties of nucleus pulposus. Spine (Phila Pa 1976) 20, 665-669 (1995).

51. Mulleman, D., Mammou, S., Griffoul, I., Watier, H. \& Goupille, P. Pathophysiology of disk-related sciatica. I.--evidence supporting a chemical component. Joint Bone Spine 73, 151-158 (2006).

52. Mebius, R. E. \& Kraal, G. Structure and function of the spleen. Nat. Rev. Immunol. 5, 606-616 (2005).

53. Risbud, M. V. \& Shapiro, I. M. Role of cytokines in intervertebral disc degeneration: pain and disc content. Nat. Rev. Rheumatol. 10, 44-56 (2014).

54. Nordic Medico-Statistical Committee. NOMESCO Classification of Surgical Procedures (NCSP) (Nordic Medico-Statistical Committee, 2011).

55. Gormley, P. et al. Meta-analysis of 375,000 individuals identifies 38 susceptibility loci for migraine. Nat. Genet. 48, 856-866 (2016).

56. Wolfe, F. et al. The American College of Rheumatology preliminary diagnostic criteria for fibromyalgia and measurement of symptom severity. Arthritis Care Res. (Hoboken) 62, 600-610 (2010)

57. Schiffman, E. et al. Diagnostic criteria for temporomandibular disorders (DC/TMD) for clinical and research applications: recommendations of the International RDC/TMD Consortium Network ${ }^{\star}$ and Orofacial Pain Special Interest Groupdagger. J. Oral Facial Pain Headache 28, 6-27 (2014).

58. Bouhassira, D. et al. Comparison of pain syndromes associated with nervous or somatic lesions and development of a new neuropathic pain diagnostic questionnaire (DN4). Pain 114, 29-36 (2005).

59. Gulcher, J. R., Kristjansson, K., Gudbjartsson, H. \& Stefansson, K. Protection of privacy by third-party encryption in genetic research in Iceland. Eur. J. Hum. Genet. 8, 739-742 (2000)

60. Gudbjartsson, D. F. et al. Large-scale whole-genome sequencing of the Icelandic population. Nat. Genet. 47, 435-444 (2015).

61. McKenna, A. et al. The Genome Analysis Toolkit: a MapReduce framework for analyzing next-generation DNA sequencing data. Genome Res. 20, 1297-1303 (2010).

62. Bulik-Sullivan, B. K. et al. LD Score regression distinguishes confounding from polygenicity in genome-wide association studies. Nat. Genet. 47, 291-295 (2015).

63. Vilhjalmsson, B. J. et al. Modeling linkage disequilibrium increases accuracy of polygenic risk scores. Am. J. Hum. Genet. 97, 576-592 (2015).

64. Gudbjartsson, D. F. et al. Sequence variants from whole genome sequencing a large group of Icelanders. Sci. Data 2, 150011 (2015).

\section{Acknowledgements}

We thank the participants, research nurses and staff at the Patient Recruitment Center, clinical data management specialists at the Landspitali University Hospital, and all clinical collaborators and colleagues who contributed to phenotype ascertainment, recruitment, collection of data, sample handling and genotyping. The financial support from the European Commission to the NeuroPain project (FP7\#HEALTH-2013-602891-2) and the National Institutes of Health (R01DE022905) is acknowledged.

\section{Author contributions}

G.B., S.B., P.S., D.G., U.T., H.S., T.E.T. and K.S. designed the study and interpreted results. G.B., U.S., T.G., A.B., I.H.O., E.U., A.V., R.H., K.O.K., T.R., I.J. and T.E.T. carried out case ascertainment. G.B., S.B., A.O., M.L.F., A.K., G.M., G.S., U.S., G.T., G.B.W., O.T.M., P.S. and D.G. performed statistical and bioinformatics analyses. G.B., S.B., T.G., A.B., I.H.O., E.U., P.S., D.G., U.T., H.S., T.E.T and K.S. drafted the manuscript. All authors contributed to the final version of the paper.

\section{Additional information}

Supplementary Information accompanies this paper at http://www.nature.com/ naturecommunications

Competing financial interests: The authors declare competing financial interest. G.B., S.B., A.O., M.L.F., A.K., G.M., U.S., G.S., G.T., G.B.W., O.T.M., U.T., P.S., D.G., H.S., T.R., I.J., T.E.T. \& K.S. are employees of deCODE Genetics/Amgen, Inc. 
Reprints and permission information is available online at http://npg.nature.com/ reprintsandpermissions/

How to cite this article: Bjornsdottir, G. et al. Sequence variant at $8 \mathrm{q} 24.21$ associates with sciatica caused by lumbar disc herniation. Nat. Commun. 8, 14265 doi: $10.1038 /$ ncomms14265 (2017).

Publisher's note: Springer Nature remains neutral with regard to jurisdictional claims in published maps and institutional affiliations. (c) (i) This work is licensed under a Creative Commons Attribution 4.0 International License. The images or other third party material in this article are included in the article's Creative Commons license, unless indicated otherwise in the credit line; if the material is not included under the Creative Commons license, users will need to obtain permission from the license holder to reproduce the material. To view a copy of this license, visit http://creativecommons.org/licenses/by/4.0/

(C) The Author(s) 2017 